\title{
A Network-Based Model for Mitigating Traffic Jams in Road Networks
}

\author{
Ahmed Salih Hasan \\ Department of Computer Science, University of Mosul, Mosul 41002 Iraq \\ Email: ahmed_salih_h@uomosul.edu.iq
}

\begin{abstract}
In recent years, the city of Mosul, which is the capital city of Nineveh province in the North of Iraq, had witnessed an unstable situation (e.g., wars, internal conflicts) that led to destructing most of the infrastructure including city roads. In addition, the population of Mosul is currently concentrated on the east coast of the city. Therefore, this situation has caused a server traffic jam and the roads have become overloaded, which is time-wasting when accessing a particular place in the city. In this analytical study, the roads of the east coast of Mosul city are modeled in the form of a Road Network. The proposed approach is based on concepts inspired from Complex Networks and their measurements such as clustering coefficient, betweenness, degree, and closeness. The dataset of this work was collected from Google Earth with the support of governmental offices and road-experienced individuals. The created network represents the road network of Mosul city. In the results, suggestions and recommendations are provided, which can contribute to alleviating the problem of traffic congestion in the city of Mosul. The provided suggestions do not need a high cost because the proposed approach benefits the current road networks with few modifications. The proposed approach is applicable to any city of interest.
\end{abstract}

Keywords: Road Networks, Complex Networks, Traffic Jam

\section{Introduction}

Today's world is witnessing a great revolution in technology. This revolution has affected our lives in many different aspects. One of the most observed aspects is the development of our cities. Some cities are considered to be highly-populated (metropolitan cities), while other cities are low populated (micropolitan cities). This specific level is determined by the size of the city and how the population is deployed in the physical environment of the city [1]. The issue in metropolitan cities is the limitation in the movements of people due to the crowded roads and the high traffic load on streets. High levels of a crowd or a day-rush-hours yield time-wasting for people [1]. Most people spend hours on roads daily making road networks a crucial part of their daily lives [2].

This search tries to investigate and study a particular case, which is the case of the east coast of Mosul city (see Fig. 1). The motivation in this study is that the east coast of Mosul city represents the most crowded area in the north of Iraq. The recent war against ISIS caused a lot of damaged places making people displaced to the less damaged areas.

To this end, this work plans to generate a complex network that contains nodes and edges [3]. Each node represents an intersection, and the edges are the streets that connect the intersections. Then, the study aims at visualizing and analyzing the generated network and comes up with facts on the network road of Mosul city. This work also tries to provide 
suggestions and recommendations that can be adopted by Mosul city officials. In the analysis, the concepts of complex networks, as well as inspiring concepts from graph theory, are involved.

According to the literature presented in Section 2, there is a lack in providing studies that consider Iraqi roads, especially the special case of Mosul city. Hence, the contribution of this work is to propose an approach that analyzed and studied the case of Mosul city roads and provides low-cost recommendations that can contribute to mitigating the traffic jams in the city.

The rest of this paper is organized as follows: Section 2 surveyed the literature, Section 3 present the research method including the dataset collection of the generated road network. Section 4 demonstrates the results alongside the discussions. Finally, this paper is concluded in Section 5.

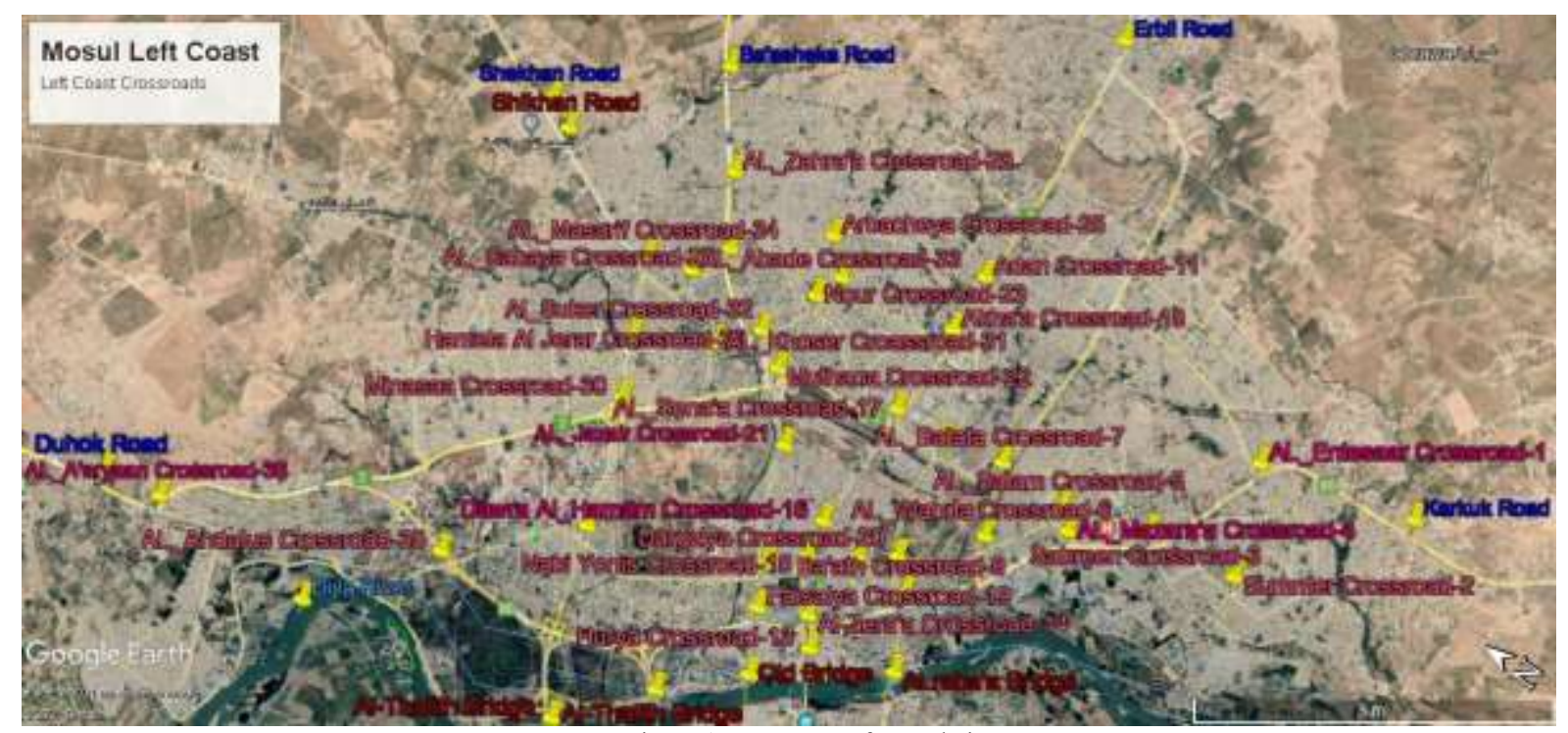

Figure 1: East coast of Mosul city.

\section{Literature Review}

Complex networks have been used in analyzing road networks. This approach is considered an efficient way for investigating road maps due to the deep relation-analysis that can be performed. However, the literature has a lack in providing researches that use complex networks concepts to provide solutions in countries such as Iraq. In this section, some works that are the closest ones to our work are presented. Tsiotas in 2020 [4] studied the interregional Greek road network by applying complex network analysis. The study aims to extract the socioeconomic information of the network topology and interpret the way in which this road network serves and promotes regional development. In another study performed by Ali et al. in 2020 [5] aimed to incorporate graph reduction and centrality interpolation while utilizing some already-efficient complex networks centrality algorithms to produce ready-to-analyze road scores for the entire given dataset while reducing the cost of analysis. Their approach was efficient to reduce the time consumed for computations when compared to the conventional algorithms that do not move from a place to another. Hu et al. in 2019 [6] proposed a generic learning framework called Graph Convolutional Weight Completion (GCWC) that exploited the topology of a road network graph to estimate the weights for all roads. Then, they incorporated contextual information into GCWC to improve accuracy. Their method was applied on data from a highway toll gate network and GPS data. Their results showed the most 
important roads based on their weight values. Moreover, Aduory et al. in 2010 [7] studied the geographic structure of the road network in a city to find out paths of transport roads and its length, density and efficiency. The road network of a city was also affected by human factors such as agricultural and industrial activities beside population distribution. In this context, the study of Awel [8] in 2007 analyzed parts of Arada city in Ethiopia. The author used geographic information systems to analyze road networks through identifying the best paths and service areas of the roads. The study found out that geographic information systems could be applied for the rest of city roads to put out needed plans to solve the problem of the city.

\section{Research Method}

\subsection{Data Collection}

One of the main parts of making roads' network for the east coast of Mosul city is the data collection process. Before initiating work, data should be collected about roads and crossroads within the east coast of the city. The data collection process needs to analyze and cover all areas and roads to specify main roads and important crowded crossroads in order to build roads' networks. The data collection was carried out with the help of experts of traffic engineering in Mosul municipality as well as the Nineveh traffic directorate. Also, the data collection process was performed with the help of road professionals as they know well all roads and crossroads that have traffic problems through surveys that include questionnaires about the name of crossroads and the degree of crowdedness, the cause of crowding, and the names of other crossroads and areas connected with. The form included columns for the name of the crossroad, which helped to define network nodes. Another column for the number of crossroads and areas connected with it, which represents node degree. Another column was for the degree of crowdedness which helped to calculate network measurements. The last column was the cause of crowding, which helped in understanding the network and providing suggestions for the problem considered in this project. As additional information that was used in the visualization was the coordinates (latitude and longitude) of each intersection based on Google Earth data. The main tools used in the data collection process were Google Earth and Google maps since they contain detailed and rich information about crossroads and their coordinates. After finishing the data collection, the stage of data entry was carried out aiming to create the dataset. Data entry was carried out through Microsoft Excel, and then the Gephi software was used to import the data to visualize the network [9]. The statistics and calculations were performed using R language [10]. The number of intersections collected in this search was 36 with 66 roads (streets) connecting them.

Moreover, the strategy followed in building the network was based on considering intersections as nodes and two intersections are connected if and only if there is a direct road between both. Each node in the network was projected based on its coordinates on the map. Therefore, the network visualization was based on real coordinates retrieved from Google Earth.

\subsection{Network Generation and Visualization}

As mentioned, the visualization of the network was performed using Gephi. The collected dataset was used for visualizing and analyzing the roads network of the east coast of Mosul city. Figure 2 depicts the visualization of the generated network. Each node represents an 
intersection in the city and the edges are the roads (streets) that connect them. In the collected dataset, intersections were classified based on a load of each intersection. The value of load can be ranged from 1 (less loaded) to 5 (heavy loaded) at city rush hours, the size of nodes reflects the load level (see Figure 2). Based on this figure, it can be seen how these intersections are connected to each other and how different loads intersections are related to each other. It can also be observed that low-level loads intersections are positioned between the high-level loads intersections. This is important to be considered as these intersections play a significant role in mitigating the crowd during rush hours. Although the coordinates of each intersection were used in the visualization, a weight is assigned to each edge in the network as an indicator of the distance between two intersections. Three levels of distances were used, 1 for short distances, 2 for medium distances, and 3 for the long distances between intersections.

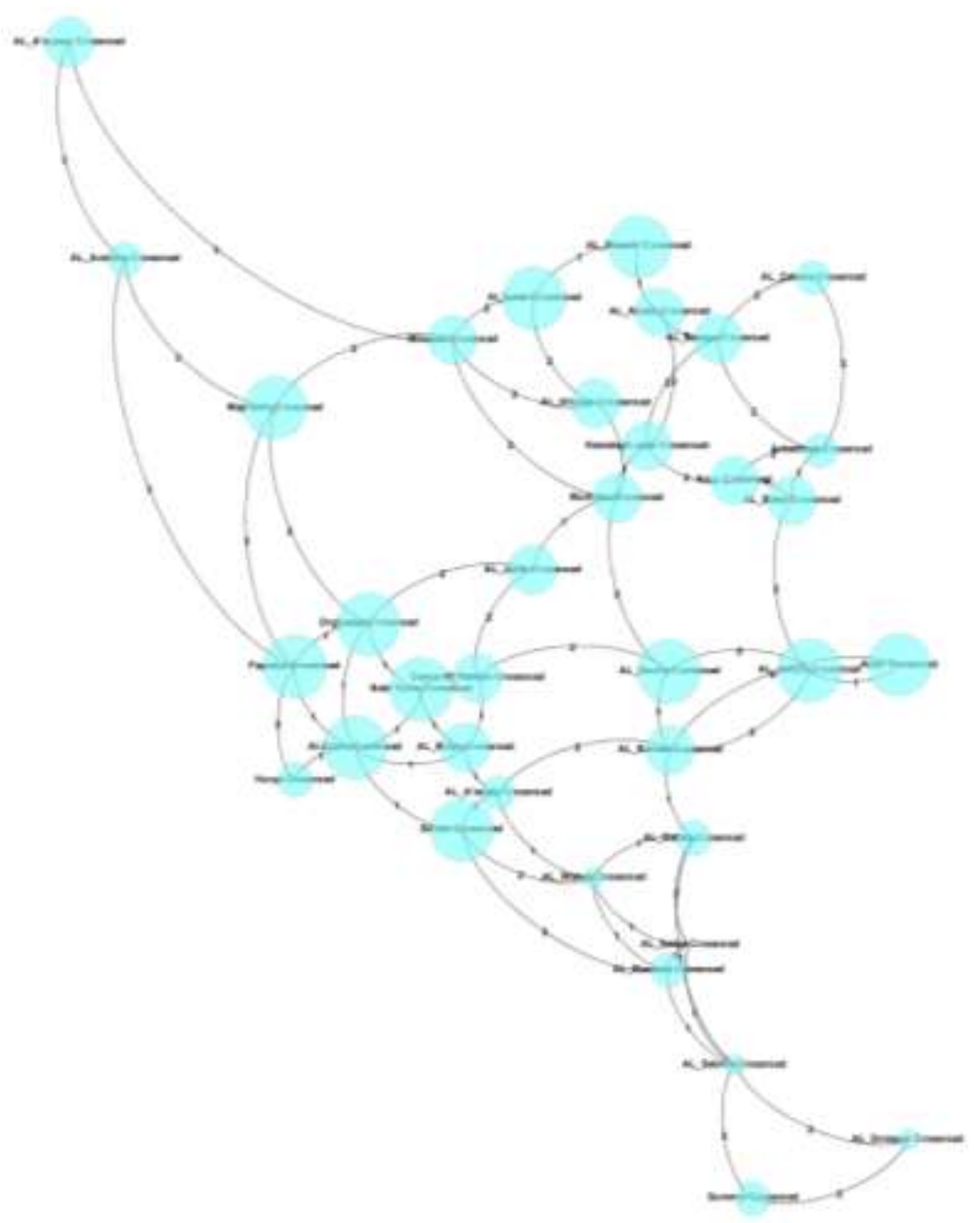

Figure 2: Mosul east coast road network, node size reflects the load of the corresponding intersection (a bigger size reflects a heavy-loaded intersection).

Figure 3 depicts, five clusters each of which reflects a group of intersections based on their loads. It can be observed that all the clusters are highly connected. The blue nodes are the most loaded intersections with a load level of 5 and represent $36.11 \%$ of the intersections. Also, the orange nodes, which reflect the next most loaded intersections with a load level of $30.56 \%$. Based on these results, the majority of the intersections on the east coast of Mosul city are heavy-loaded and represent $66.67 \%$ of the total intersections in the city. 


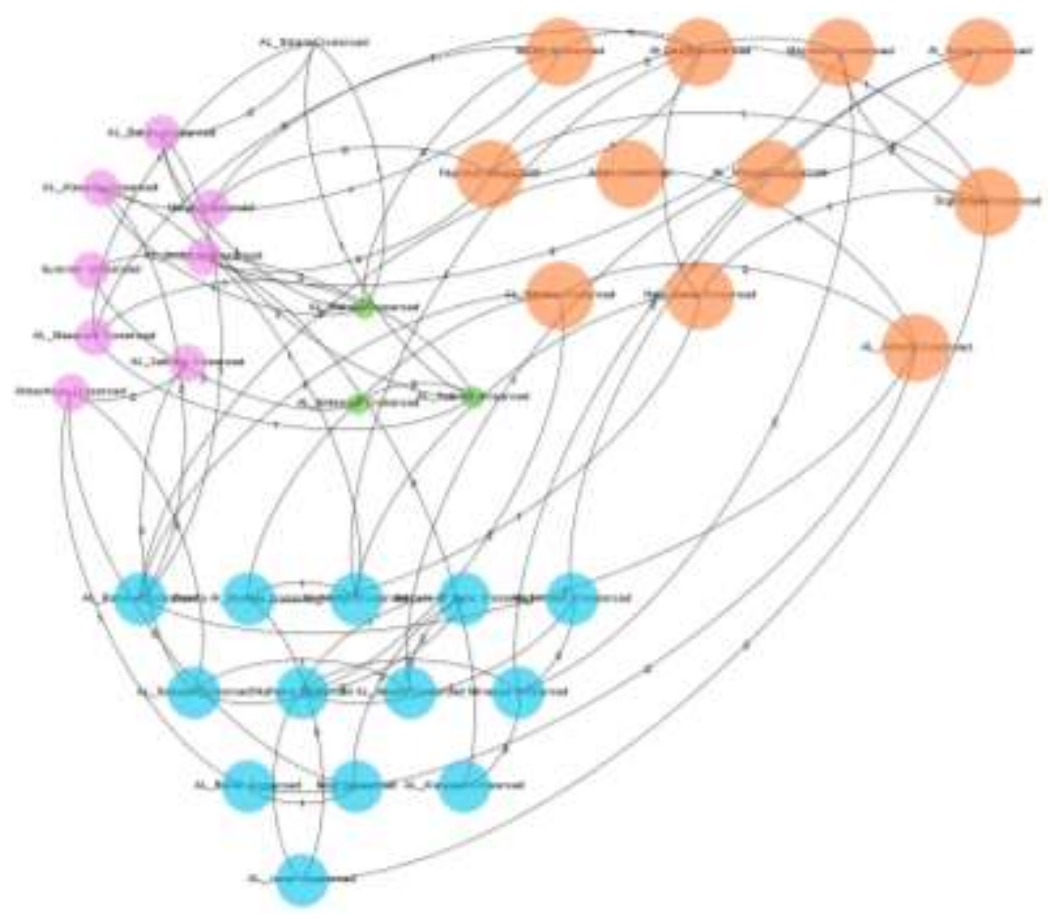

Figure 3: Mosul east coast intersections visualization based on the load of each intersection.

\section{Results and Discussion}

\subsection{Network Characteristics}

Based on the generated roads network, the main characteristics of the network were calculated as presented in Table 1. The average degree of 3.667 is considered low since it reflects that intersections are weakly connected, which is not desired in road networks. The diameter of the network is also not sufficient because it reflects a high distance between the farthest regions on the east coast of the city. The density of intersections is acceptable compared to other networks in the literature. Moreover, the average clustering coefficient [11] is also weak and reflects a low tendency of the roads to cluster together. The other feature is the average path length [12][13], which is acceptable since it means that less than 3 roads are needed to access any region in the city. In fact, the general characteristics of the network are not sufficient in terms of the aforementioned measurements compared to similar networks in the literature. However, we still struggle moving into the city during the rush hours. Here, we see that the roads of the city need to be exploited more professionally.

Table 1: The characteristics of Mosul city east coast roads

\begin{tabular}{ccccccc}
\hline of Nodes \# & of Edges \# & Average Degree & Diameter & Density & $\begin{array}{c}\text { Average Clustering } \\
\text { Coefficient }\end{array}$ & $\begin{array}{c}\text { Average Path } \\
\text { Length }\end{array}$ \\
36 & 66 & 3.667 & 8 & 0.105 & 0.394 \\
\hline
\end{tabular}

\subsection{Network Measurements}

According to the description of the previous section, more analysis is needed to be performed using network measurements as follows:

\subsubsection{Betweenness Centrality Measurement.}

The betweenness centrality measurement is the number of times that a node appears in the shortest path of the other pairs in the network. In other words, it reflects how influential a node is or how well-positioned a node is within a network [14]. The betweenness centrality was 
calculated for all the intersections in the network. The intersections were ranked based on their values of betweenness centrality in a descending order (considering the highest 10 because after that the level of betweenness is significantly decreased). Table 2 presents these values and it is clear that the Muthana intersection has the highest betweenness centrality. This means that most people in the regions around Muthana square pass-through this intersection. This intersection has a small capacity compared to its importance. The other intersections in this list need to be improved. For instance, widening the streets of these intersections or putting more control on them. Also, the officials can adopt a transformation strategy that transforms an intersection into a regular street. Figure 4 demonstrates the visualization of the Mosul city east coast road network. In this visualization, node size is driven by its corresponding betweenness. Different colors reflect different loads in the intersection node. It is clear that the Muthana intersection is the most well-positioned in the network. It should be mentioned that betweenness and load are not similar since the betweenness reflects a fact about the position. Therefore, although Muthana is not classified as the highest loaded (load $=4)$ intersection, but its position makes this intersection important and can be utilized more feasibly.

Table 2: Top 10 intersections in terms of betweenness centrality (influential and well-positioned intersections)

\begin{tabular}{|c|c|c|c|}
\hline Rank & Intersections & Betweenness & Load \\
\hline $1^{\text {st }}$ & Muthana & 0.3129 & 4 \\
\hline $2^{\text {nd }}$ & Barawe & 0.2601 & 5 \\
\hline $3^{\text {rd }}$ & Sena'a & 0.2234 & 5 \\
\hline $4^{\text {th }}$ & Zera'a & 0.1622 & 4 \\
\hline $5^{\text {th }}$ & Hamela Al Jerar & 0.1579 & 3 \\
\hline $6^{\text {th }}$ & AlBatata & 0.1504 & 4 \\
\hline $7^{\text {th }}$ & Minasa & 0.1296 & 5 \\
\hline $8^{\text {th }}$ & AlAkha'a & 0.1232 & 5 \\
\hline $9^{\text {th }}$ & Ba'ath & 0.2592 & 2 \\
\hline $10^{\text {th }}$ & Sabren & & \\
\hline
\end{tabular}

\subsubsection{Weighted Degree Centrality.}

The weighted degree centrality shows how many roads can be reached using a particular intersection [15]. Table 3 shows Al-Zera'a intersection has the highest connections and leads to most of the intersections in Mosul city followed by the Muthana intersection. In fact, as we believe, the best strategy when dealing with such intersections is to provide some direct/indirect shortcuts from this kind of intersection to the other intersections. This contributes to distributing the loads to the heavily loaded intersections and obtains an even way to balance the loads. It can also be observed that the Wahda intersection has a high degree with a low level of load of 2. This feature should be exploited since this intersection may solve a lot of traffic issues. For instance, a shortcut can be established to, for example, Adan intersection or Al-Akha'a intersections. Figure 5 demonstrates the visualization where the node size reflects the degree of the corresponding intersection. 
AL_A'aryaan Crossroad

AL_Andalus Crossroad

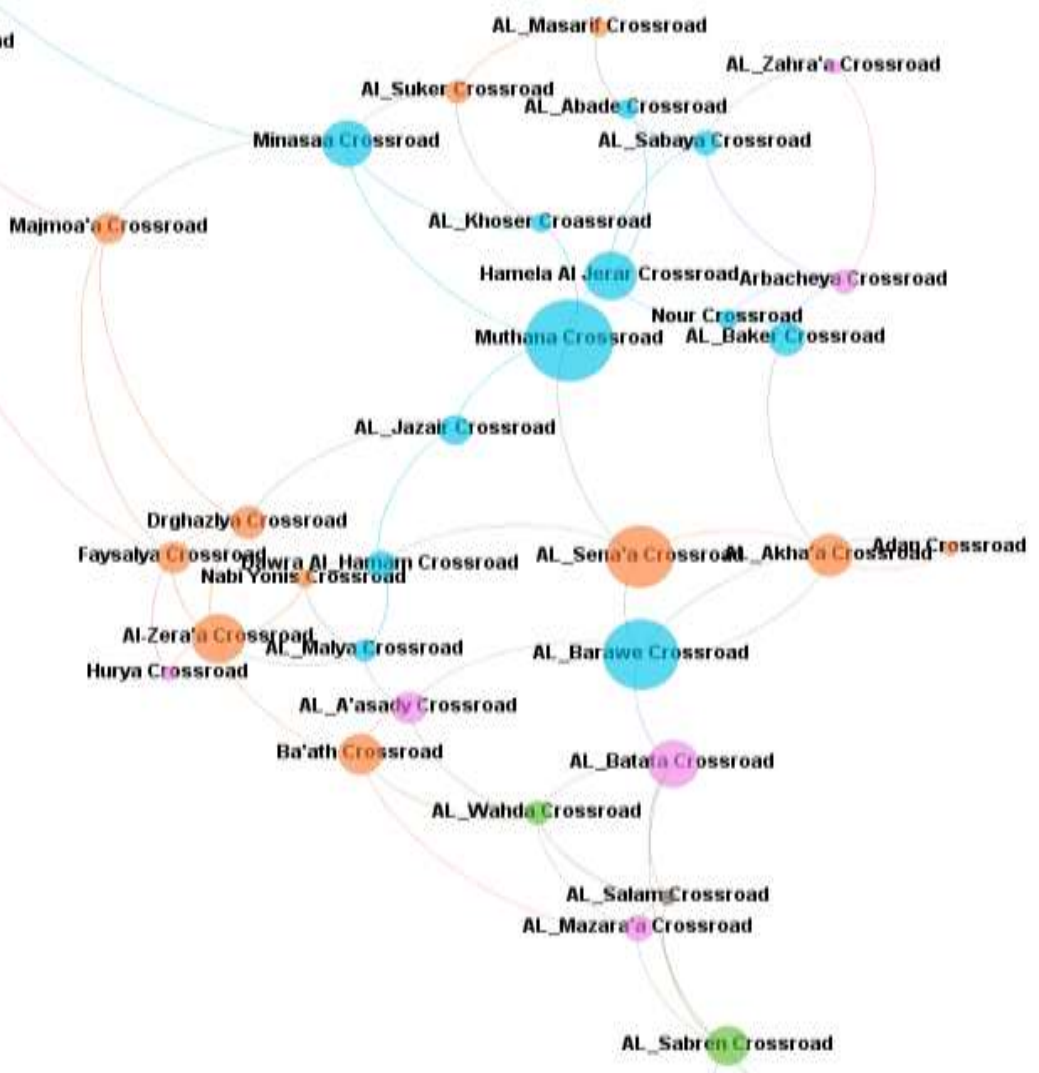

AL_Entesaas Crossroad

Summer crossroad

Figure 4: Betweenness-Based visualization of east coast network of Mosul city.

Table 3: Top 10 intersections in terms of weighted degree centrality (highly connected).

\begin{tabular}{|c|c|c|c|}
\hline Rank & Intersections & Degree & Load \\
\hline $1^{\text {st }}$ & Zera'a & 6 & 5 \\
\hline $2^{\text {nd }}$ & Muthana & 5 & 4 \\
\hline $3^{\text {rd }}$ & Barawe & 5 & 4 \\
\hline $4^{\text {th }}$ & Minasaa & 5 & 2 \\
\hline $5^{\text {th }}$ & Sabreen & 5 & 5 \\
\hline $6^{\text {th }}$ & Drghazlya & 5 & 5 \\
\hline $7^{\text {th }}$ & Faysalya & 5 & 2 \\
\hline $8^{\text {th }}$ & Wahda & 4 & 5 \\
\hline $9^{\text {th }}$ & Sena'a & 4 & 4 \\
\hline $10^{\text {th }}$ & Hamela Al Jerar & & \\
\hline
\end{tabular}




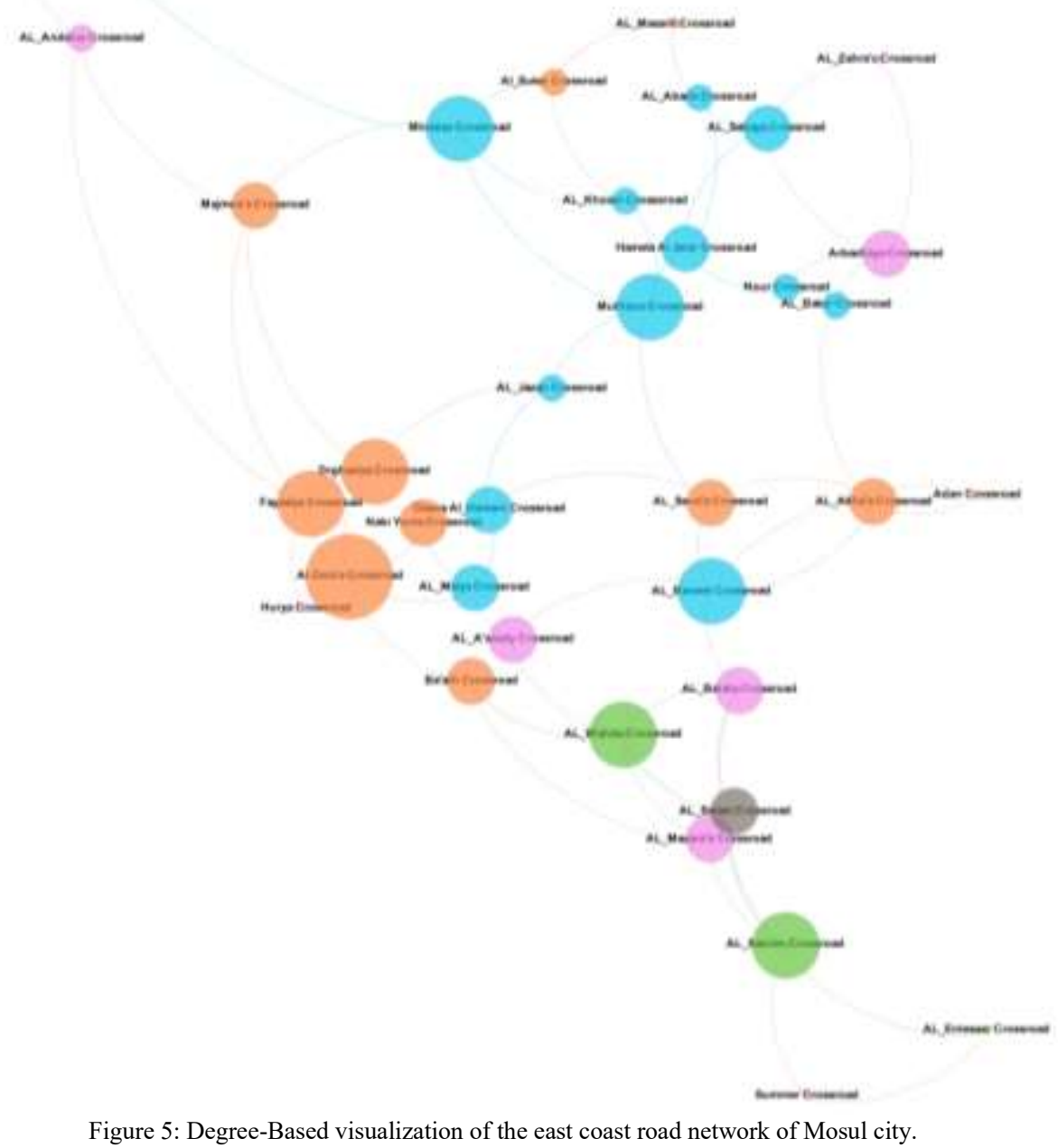

\subsubsection{Closeness Centrality.}

The closeness centrality reflects how close an intersection is to the other streets and intersections in the city [16]. Table 4 presents the intersections based on their closeness centrality values. Based on this table, it is clear that some of the intersections are not given much attention by the officials. These intersections need to have more control to mitigate the loads on the main intersections in the city. The Sena'a intersection is considered the closest intersection to all the intersections on the east coast of Mosul city. This feature could be exploited by widening its streets or its leading streets aiming to contain more capacity. Figure 6 shows the visualization of the network. Node size reflects the level of closeness centrality (bigger nodes have the highest closeness centrality). The visualization and the table also tell us the other intersections that are considered very close to other intersections in the road network of the east coast of Mosul city. 
Table 4: Top 10 intersections in terms of closeness centrality (closest to all roads).

\begin{tabular}{|c|c|c|c|}
\hline Rank & Intersections & Closeness & Load \\
\hline $1^{\text {st }}$ & Sena'a & 0.3684 & 5 \\
\hline $2^{\text {nd }}$ & Muthana & 0.3645 & 4 \\
\hline $3^{\text {rd }}$ & Barawe & 0.3500 & 5 \\
\hline $4^{\text {th }}$ & Akha'a & 0.3240 & 4 \\
\hline $5^{\text {th }}$ & Jazair & 0.3240 & 5 \\
\hline $6^{\text {th }}$ & Zera'a & 0.3211 & 5 \\
\hline $7^{\text {th }}$ & Dawra Al-Hamam & 0.3211 & 4 \\
\hline $8^{t h}$ & Drghazlya & 0.3211 & 4 \\
\hline $9^{t h}$ & Minasaa & 0.3181 & 3 \\
\hline $10^{t h}$ & A'asady & 0.3181 & 5 \\
\hline
\end{tabular}

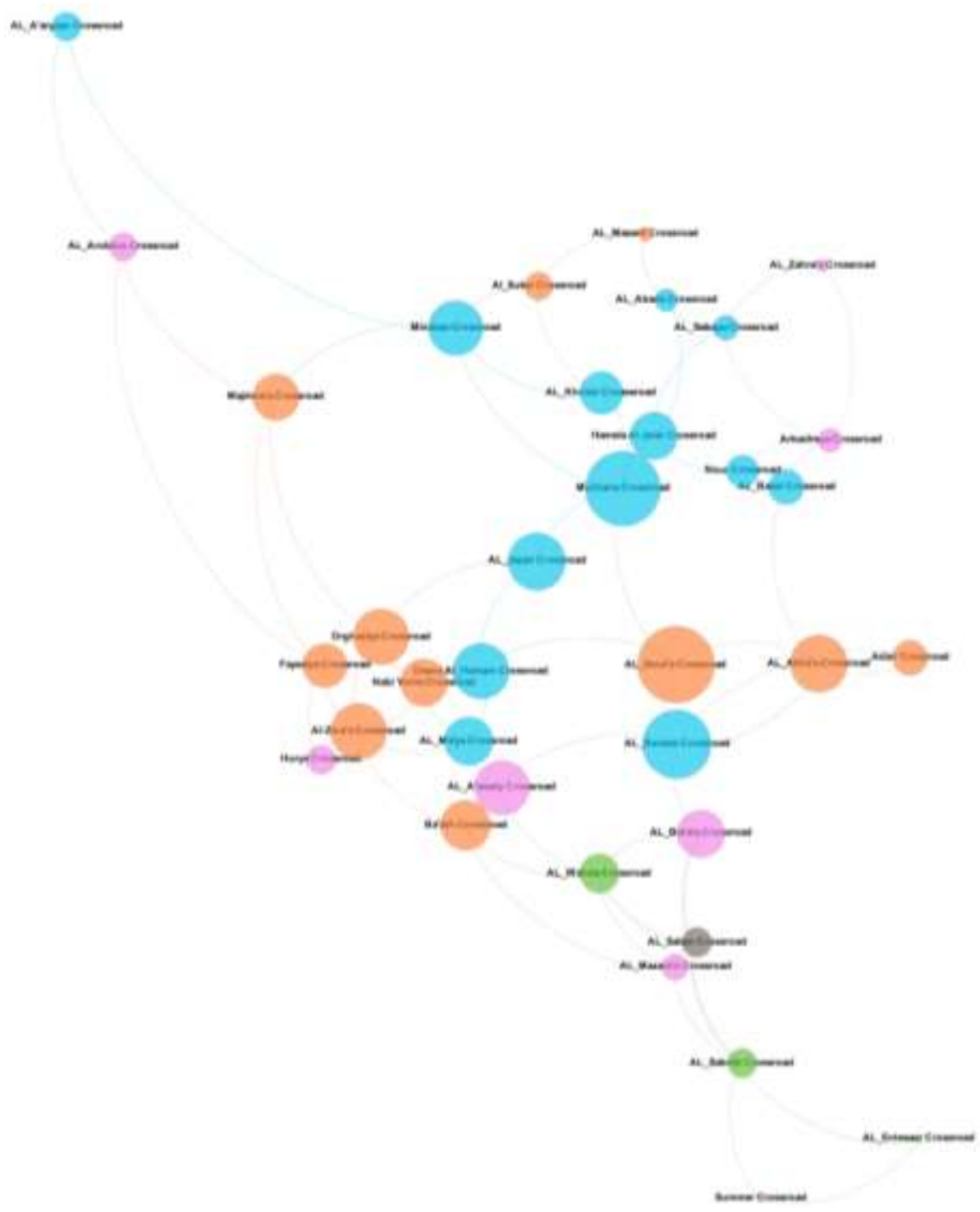

Figure 6: Closeness-Based visualization of the east coast road network of Mosul city. 


\subsubsection{Intersections to be Considered}

Based on the aforementioned results, the collective analysis can be utilized. In other words, this work uses network measurements to have indicators on the generated network. Each measurement reflects a particular fact about the network structure. Therefore, the collective reflection of the measurements on the network is used to come up with concrete analysis.

Given that we have the three tables 2, 3, and 4, the intersections among these tables are considered. The goal of this step is to have a list of intersections that are structurally important in the network. Table 5 presents the intersections that should be given more attention when improving the road network of Mosul city. These intersections are extracted from the network and visualized in Figure 7. Interestingly, it can be seen that 4 of them are already connected, which makes the improvements easier.

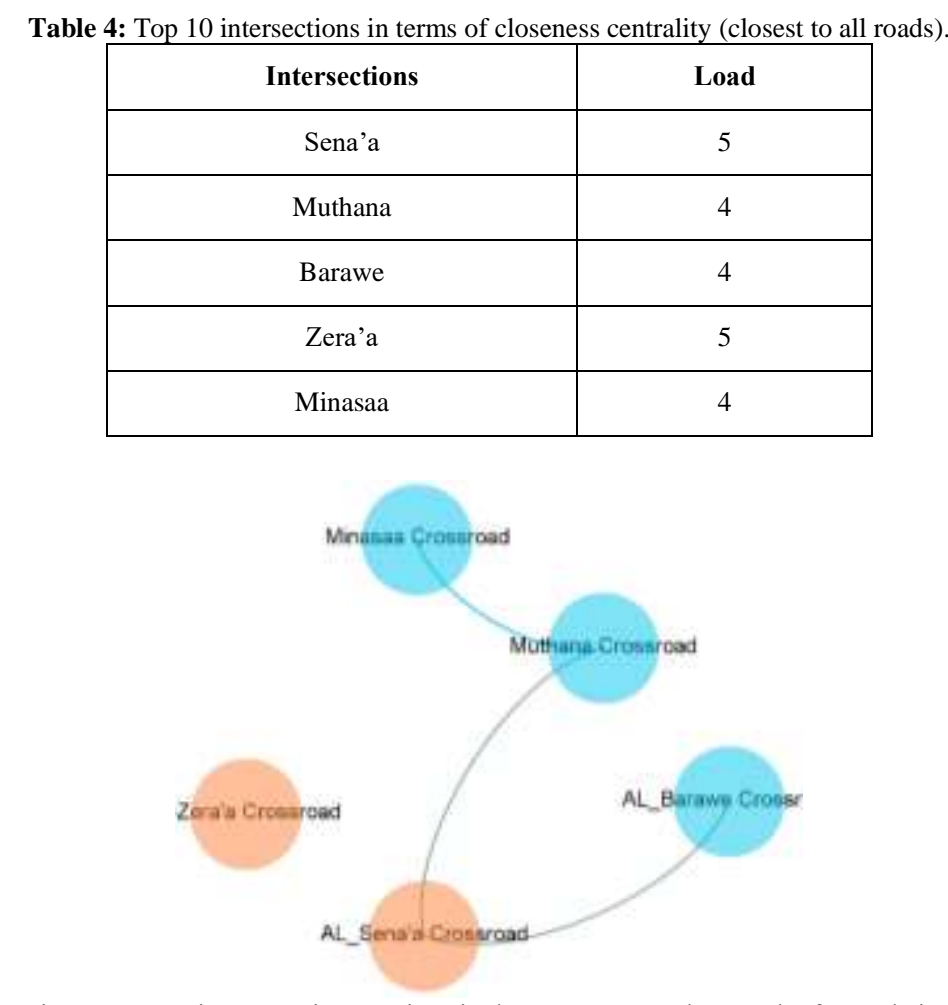

Figure 7: Most important intersections in the east coast road network of Mosul city.

Based on the results in the previous sections, the work can be summarized by the following facts and recommendations:

- The complex network approach can be considered a powerful tool in investigating and analyzing road networks.

- Adopting some shortcuts among these the highest-betweenness (see Table 2) centrality intersections can improve the traffic flow in the city.

- Based on the values of betweenness centrality of the intersections, it can be inferred that some intersections with less load can play a significant role in mitigating the traffic jam that happens during rush hours. This exact fact leads us to conclude that when having full control of this kind of intersection, the overall traffic load will significantly decrease. In addition, these intersections can be improved in terms of widening their streets in a way that does not cost a lot from the province. 
- Connecting/shortcutting the intersections with the highest betweenness centrality leads the overall betweenness to be increased, which eventually makes the traffic more flexible and the time consumed to get to a particular destination will decrease.

- The closeness centrality measurements reflect the fact that some important intersections are not well-connected. This leads to having long distances to get to a destination. We believe that the best strategy that can be adopted is to connect the intersection with the lowest values of closeness with each other. This process will increase the values of the closeness of the intersections and make people use different routes to get to their destinations.

- A general strategy for the road network of Mosul city is to provide some direct/indirect shortcuts among the intersections. This can contribute to enhancing the general structure of the network and make it closer to being fully connected as well as increase the average clustering coefficient of the network, which is desired. Also, this strategy can contribute to distributing the loads to the heavily loaded intersections and obtain an even way to balance the loads.

- All the recommendations and suggestions in this project do not need to establish or build new streets or new intersections; they are most about enhancing the current structure of the network to have better traffic performance and save the time consumed by people to get their work, schools, companies, etc.

\section{Conclusions}

In this paper, the road networks of the east coast of Mosul city were modeled using concepts inspired from complex networks. The dataset that was used in this project were carefully collected from Google Earth and Google Maps as well as road experts. Then, the collected dataset was used to generate the Road Network of the east coast of Mosul city. The intersections were represented as nodes and the edges among them represented city streets. The generated network was visualized and analyzed using network measurements such as betweenness centrality, closeness centrality, and degree centrality. The obtained results were used to come up with low-cost recommendations that can contribute to improving the structure of the road network and eventually reduce the time consumed for people to get to their destination as early as possible.

As future work, we plan to investigate the road networks of Mosul city using the same approach that was involved in this work. This kind of investigation can support having a wider view of the roads in Mosul. Moreover, investigating the giant component of the roads will enrich the officials with more useful information and help them in providing better strategies that lead to having more flexible solutions to the traffic jam issue.

\section{Acknowledgment}

We would like to thank the University of Mosul and Mosul Traffic directorate for all the support in making this work achieved.

\section{References}

[1] Mahmood, B., \& Menezes, R. (2016). The role of human relations and interactions in designing memory-related models for sensor networks. Sensors \& Transducers, 199(4), 42-51.

[2] Ren, Y., Cheng, T., \& Zhang, Y. (2019). Deep spatio-temporal residual neural networks for road- 
network-based data modeling. International Journal of Geographical Information Science, 33(9), 1894-1912.

[3] Mahmood, B., Tomasini, M., \& Menezes, R. (2015, October). Estimating memory requirements in wireless sensor networks using social tie strengths. In 2015 IEEE 40th Local Computer Networks Conference Workshops (LCN Workshops) (pp. 695-698). IEEE.

[4] Tsiotas, D. (2020). Modeling of the Greek road transportation network using complex network analysis. arXivpreprint arXiv:2003.08091.

[5] Ali, A., Chen, Y., Fuller, D., \& Al-Eidi, S. (2020, February). Road Importance Using ComplexNetworks, Graph Reduction \& Interpolation. In 2020 International Conference on Computing, Networking andCommunications (ICNC) (pp. 855-859). IEEE.

[6] Hu, J., Guo, C., Yang, B., \& Jensen, C. S. (2019, April). Stochastic weight completion for road networks using graph convolutional networks. In 2019 IEEE 35th International Conference on Data Engineering(ICDE) (pp. 1274-1285). IEEE.

[7] Aduory, R. (2010). Geographical Analysis of the Network of Roads in the Duor for 2008, Journal of Tikrit University for the humanities, University of Tikrit, Iraq, 17(3).

[8] Awel, J. (2007). GIS-Based Road Network Analysis in The Sub City of Arada (Doctoral dissertation, Addis Ababa University).

[9] Bastian, Mathieu; Heymann, Sebastien; Jacomy, Mathieu (2009), Gephi: An Open Source Software for Exploring and Manipulating Networks, AAAI Publications, Third International AAAI Conference on WeblogandSocial Media, retrieved 2011-11-22

[10] Fox, John \& Andersen, Robert (January 2005). Using the R Statistical Computing Environment to Teach Social Statistics Courses, Department of Sociology, McMaster University. Retrieved 2006-08-03.

[11] Mahmood, B., Tomasini, M., \& Menezes, R. (2015, February). Social-based Forwarding of Messages in Sensor Networks. In SENSORNETS (pp. 85-90).

[12] Lan, T., Li, Z., \& Zhang, H. (2019). Urban allometric scaling beneath structural fractality of road networks. Annals of the American Association of Geographers, 109(3), 943-957.

[13] Mahmood, B., \& Menezes, R. (2013, April). United states congress relations according to liberal and conservative newspapers. In 2013 IEEE 2nd Network Science Workshop (NSW) (pp. 98-101). IEEE.

[14] Mahmood, B. M., Sultan, N. A., Thanoon, K. H., \& Khadhim, D. S. (2020). Collaboration Networks: University of Mosul Case Study. AL-Rafidain Journal of Computer Sciences and Mathematics, 14(1), 117-133.

[15] Skiens, S. Implementing Discrete Mathematics: Combinatorics and Graph Theory with Mathematica. Reading, MA: Addison-Wesley, 1990.

[16] Mahmood, B., Tomasini, M., \& Menezes, R. (2015). Social-driven information dissemination for mobile wireless sensor networks. Sensors \& Transducers, 189(6), 1-11. 